\title{
Meta
}

Journal des traducteurs

Translators' Journal

\section{Depecker, Loïc (dir.) (1997) : La mesure des mots. Cinq études d'implantation terminologique, Rouen, Publications de l'Université de Rouen, XXXVII + 528 p.}

\section{Aline Francoeur}

Volume 43, numéro 2, juin 1998

URI : https://id.erudit.org/iderudit/002770ar

DOI : https://doi.org/10.7202/002770ar

Aller au sommaire du numéro

Éditeur(s)

Les Presses de l'Université de Montréal

ISSN

0026-0452 (imprimé)

1492-1421 (numérique)

Découvrir la revue

Citer ce compte rendu

Francoeur, A. (1998). Compte rendu de [Depecker, Loïc (dir.) (1997) : La mesure

des mots. Cinq études d'implantation terminologique, Rouen, Publications de

l'Université de Rouen, XXXVII + 528 p.] Meta, 43(2), 314-318.

https://doi.org/10.7202/002770ar d'utilisation que vous pouvez consulter en ligne.

https://apropos.erudit.org/fr/usagers/politique-dutilisation/ 


\section{DOCUMENTATION}

\section{Comptes rendus}

- DEPECKER, Loïc (dir.) (1997) : La mesure des mots. Cinq études d'implantation terminologique, Rouen, Publications de l'Université de Rouen, XXXVII + 528 p.

Les études d'implantation terminologique présentées dans La mesure des mots ont été réalisées à la demande de la Délégation générale à la langue française (DGLF) par cinq équipes de chercheurs universitaires. En commandant ces études, la DGLF souhaitait «évaluer l'impact des décisions prises en France par les commissions ministérielles de terminologie» (p. VII). Il s'agissait là d'un précédent, puisque l'usage réel des termes traités et proposés par ces commissions ministérielles n'avait jamais auparavant fait l'objet d'une étude scientifique.

Les équipes de recherche ont bénéficié de la plus grande liberté dans la réalisation de leur étude, chacune élaborant sa propre démarche méthodologique et appliquant ses propres critères d'analyse. C'est ainsi, d'ailleurs, que chacune s'est retrouvée confrontée à des problèmes particuliers, le problème central étant le suivant : quand peut-on considérer qu'un terme est implanté ? C'est dans la diversité des méthodes adoptées et, en conséquence, des résultats obtenus, que réside toute la richesse de l'ouvrage; les avenues explorées sont nombreuses et les voies de réflexion sur la question de l'implantation terminologique le sont tout autant.

La première étude d'implantation présentée dans le recueil a été réalisée sous la direction du regretté Louis Guespin par le Groupe de recherche en terminologie de l'Université de Rouen et elle porte sur 41 termes du domaine du génie génétique parus dans l'arrêté ministériel du 14 novembre 1990. Comme l'expliquent les chercheurs, l'étude s'inscrivait dans le cadre de réflexions sur la sociolinguistique, l'éditologie et la glottopolitique et fut «l'occasion d'un travail de terrain et d'une mise en œuvre des principes méthodologiques de la socioterminologie» (p. 5). Des sources écrites et orales ont servi de base à la réalisation de l'étude. Au nombre des sources écrites, on compte des dictionnaires spécialisés, des quotidiens, des revues de vulgarisation de même que des ouvrages spécialisés, des polycopiés de cours et des thèses. Quant aux sources orales, elles comprennent des enregistrements de cours et de séminaires de même que deux entretiens semi-dirigés avec des chercheurs œuvrant dans le domaine.

Le cadre théorique de l'enquête est bien situé, mais certains détails méthodologiques nous échappent, faute de précisions; on peut en fait reprocher aux auteurs de cette étude un manque de rigueur dans la présentation des données. Les résultats sont difficilement interprétables compte tenu, entre autres, que l'on ne fournit pas au lecteur la liste des 41 termes

Meta, XLIII, 2, 1998 
officiels traités. La lecture des chapitres 3, 4 et 5 de l'étude, dans lesquels on expose et commente les choix terminologiques des auteurs consultés et des répondants interrogés, est parfois ardue. On cherche en vain à deviner lesquels parmi les termes cités en exemple correspondent aux formes officialisées par les arrêtés de terminologie. Comme l'observe Loïc Depecker dans l'introduction de l'ouvrage, cette étude «livre une appréciation globale et ne permet pas de statuer véritablement sur l'implantation de chacun des termes» (p. XIII).

Par ailleurs, bien que les auteurs de l'étude précisent que «rien ne vise à évaluer le travail effectué au sein des commissions [de terminologie]» (p. 4), bon nombre des remarques du chapitre de conclusion vont dans ce sens. Ainsi, on peut lire qu'«un calcul stratégique de l'ensemble du paradigme de construction (dérivation et composition) devrait précéder le lancement d'une proposition d'équivalence pour un terme de base à franciser» (p. 33). Les chercheurs estiment de plus que les chances de réussite d'implantation du terme devraient représenter un critère déterminant à prendre à compte, et que «seules des propositions de termes faciles à retenir et faciles à manipuler par dérivation et composition auront quelques chances» (p. 34). Également, constatant que les spécialistes du génie génétique ne connaissent pas les travaux de normalisation, les auteurs de l'étude soutiennent que la publication de listes de termes recommandés n'est pas suffisante pour permettre leur implantation et qu'il faudrait plutôt exposer clairement aux destinataires les données du problème, à savoir quels sont les termes en concurrence, quelles sont les solutions possibles, pourquoi privilégie-t-on tel terme plutôt que tel autre, etc. Finalement, les chercheurs estiment qu'«en parallèle au travail in vitro de la commission, un gros travail doit être mené sur le terrain» (p. 38), ce qui permettrait d'évaluer les chances de succès des termes proposés, mais surtout de recueillir des formes produites spontanément par les locuteurs, formes qui, de leur avis, possèdent les meilleures chances de succès du fait qu'elles ont déjà une existence à tout le moins locale.

La deuxième étude, menée sous la direction de Philippe Thoiron par une équipe du Centre de recherche en terminologie et traduction de l'Université Lumière Lyon-2, porte sur 31 termes des domaines de la santé et de la médecine relevés dans les arrêtés ministériels du 2 janvier 1975 et du 7 décembre 1978. Ici, pour répondre à la demande de la DGLF, on a conçu le travail comme une étude de faisabilité et on a porté une attention particulière aux questions d'ordre méthodologique. Le degré d'implantation des termes a été mesuré à partir d'un corpus écrit et d'entretiens semi-dirigés. Le corpus écrit (249 titres) a été constitué dans une vision diachronique, en tenant compte de la variété des domaines et du degré de spécialisation des textes. L'étude de corpus a permis, d'une part, de relever et de dénombrer les occurrences des termes officialisés et, d'autre part, de procéder à la recherche de termes concurrents (anglais ou français) et à une analyse de contextes. Les données tirées de l'étude de corpus ont été cotées afin d'apprécier de façon quantitative l'emploi des termes officialisés. La cotation est basée sur divers critères, notamment la fréquence du terme officialisé dans le corpus, la présence et la fréquence de termes concurrents et l'adéquation du terme officialisé et de la définition.

Les entretiens semi-dirigés ont été conduits auprès de 30 spécialistes, 10 généralistes et 20 étudiants en médecine. Pour tester la connaissance du terme officialisé auprès des répondants, quatre situations ont été enregistrées : le répondant 1) a retrouvé le terme officialisé à partir de la définition; 2) a retrouvé le terme officialisé à partir de son correspondant anglais; 3) n'a pas reconnu le terme officialisé; 4) n'a pas retrouvé ni reconnu le terme officialisé. Une cote a été attribuée à chaque situation, ce qui a permis de représenter sous forme de moyennes la connaissance des termes par les répondants. La mesure finale utilisée pour quantifier l'implantation des termes officiels correspond à une moyenne établie à partir de l'emploi des termes dans le corpus et de l'emploi des termes par les répondants.

Les résultats de l'étude de Philippe Thoiron et de ses collègues sont présentés avec clarté et précision. Chaque terme traité fait l'objet d'un paragraphe dans lequel on décrit les 
principaux résultats de l'étude de corpus et des entretiens. Ainsi, on apprend, par exemple, que le terme clairance n'était guère employé à l'écrit avant 1984 (c'est plutôt l'usage de l'équivalent anglais clearance qui prévalait), mais que la situation s'est inversée à partir de 1985. À l'oral, clairance se retrouve souvent dans les groupes interrogés (moyenne de connaissance du terme par les locuteurs de 8,35 sur 10).

On propose à la suite des résultats une discussion des plus intéressantes sur la variabilité du degré d'implantation des termes, phénomène s'expliquant par diverses raisons qui, comme le soulignent les auteurs de l'étude, «ne sont pas toutes faciles à identifier» (p. 67). Philippe Thoiron et ses collègues ont néanmoins tenté une analyse des caractéristiques morphologiques et sémantiques des termes étudiés en vue de tirer certaines conclusions quant à leur degré d'implantation. Ils ont constaté, entre autres, que les termes officiels bien motivés et sans concurrents anglais (par exemple coloscope) se sont généralement bien implantés, tout comme ceux dont la forme anglaise phonique ou graphique est assez proche (clairance pour clearance et moniteur pour monitor, par exemple). Mais il est difficile de formuler des règles pour expliquer ce phénomène de variation de l'implantation, puisque, comme l'observent les auteurs de l'étude, «pour l'implantation des termes, comme pour d'autres faits linguistiques, l'irrationnel sait parfois trouver sa place» (p. 72).

La troisième étude a été réalisée par le Centre de linguistique et de dialectologie de l'Université de Toulouse-le-Mirail sous la direction de Jean-Louis Fossat et porte sur 59 termes du domaine de la télédétection aérospatiale. Considérant que l'implantation des termes n'est mesurable qu'à travers un ensemble de situations de communication auxquelles sont confrontés les spécialistes du domaine, les chercheurs ont établi une typologie de 22 situations de communication (orales et écrites, formelles et informelles), allant de la soutenance de thèse au cours universitaire en passant par l'article scientifique et la correspondance privée entre collègues. La démarche adoptée se distingue de celles qui ont été précédemment décrites. Ici, on a fait reposer l'enquête non pas sur l'attestation des termes dans un corpus, mais plutôt sur les déclarations d'utilisation des termes faites par deux catégories de répondants : 12 professeurs ou chercheurs dans le domaine et huit spécialistes œuvrant à l'élaboration ou à la commercialisation des produits. Les répondants ont d'abord été invités, par le biais d'un questionnaire écrit, à se prononcer sur leur conduite langagière. Ils devaient ensuite, dans les cas de rejet du terme proposé ou dans les cas d'hésitation, mentionner les termes qu'ils privilégiaient, ce qui correspond à une situation de substitution. À partir des quatre types de conduite relevés (utilisation du terme, rejet du terme, hésitation et substitution), les réponses ont été représentées quantitativement par des pourcentages illustrant les taux d'utilisation des termes officiels. Par ailleurs, des rencontres individuelles avec les répondants ont permis aux chercheurs de recueillir des données qualitatives et argumentatives plus détaillées. Les résultats de l'enquête, présentés de façon synthétique sous forme de tableaux, montrent que selon les taux d'utilisation recensés, 30 des termes traités sont bien implantés ou en bonne voie de l'être, 17 sont menacés dans leur processus d'implantation et 12 sont considérés «en danger».

Il faut de plus ajouter que les auteurs se sont intéressés d'une façon particulière aux propositions de termes émises par les répondants, ce qui leur a permis d'isoler trois grands types de comportements de substitution : l'économie linguistique, la redésignation partielle et la redésignation totale. Finalement, on propose au lecteur une analyse poussée de 10 des 59 termes étudiés dans laquelle on résume les résultats obtenus en plus de reproduire les principaux commentaires émis par les répondants.

Il ressort de l'étude dirigée par Jean-Louis Fossat que le discours des spécialistes de la télédétection aérospatiale est empreint d'une grande variabilité lexicale, qu'il résiste bien à l'emprunt et qu'il «tend vers un équilibre, soit en recherchant des solutions néologiques originales, soit en adhérant de façon mesurée sous forme de calques aux désignations angloa- 
méricaines» (p. 128). Par ailleurs, les auteurs reconnaissent avoir eu de la difficulté à vérifier l'implantation d'un certain nombre de termes récents, compte tenu de l'impossibilité, dans de tels cas, de procéder à une étude diachronique.

La quatrième étude d'implantation présentée dans La mesure des mots porte sur 41 termes du domaine de l'audiovisuel et de la publicité publiés dans les arrêtés ministériels du 24 janvier 1983 et du 10 octobre 1985. Elle a été menée par Michel Chansou, du laboratoire Lexicométrie et textes politiques, en collaboration avec l'Institut national de la langue française, le Centre national de recherche scientifique et l'École normale supérieure de Fontenay/Saint-Cloud. Cette étude prend la forme d'une observation systématique de l'usage écrit à différents niveaux de communication. Le corpus utilisé, que l'auteur décrit avec force détails, a été constitué à partir de sources variées : dictionnaires généraux et spécialisés, banques de terminologie, documents administratifs, manuels techniques, ouvrages de vulgarisation, documents pédagogiques, presse écrite générale et spécialisée, etc. Michel Chansou n'a pas tenté de mesurer quantitativement l'implantation des termes. Il propose plutôt, pour chaque terme traité, une notice détaillée qui rend compte avec une grande clarté des divers usages observés. Ainsi, on apprend entre autres que le terme remue-méninges est absent du Petit Robert 1992 et des dictionnaires techniques consultés, mais qu'il apparaît dans le Petit Larousse 1993 et le Robert \& Collins 1991. Par ailleurs, ce terme n'a été recensé dans aucun texte officiel ou administratif, pas plus que dans les manuels techniques et pédagogiques et les ouvrages de vulgarisation. Le terme remue-méninges est cependant utilisé par la presse écrite grand public, notamment dans Le Monde, Le Monde Affaires et Le Nouvel Observateur.

Dans le bilan qu'il dresse à la fin de son étude, Michel Chansou reconnaît que la présentation de données quantitatives serait intéressante, mais qu'«une telle évaluation est difficile à établir de façon significative» (p. 164). Il faut souligner que Michel Chansou innove en recourant aux dictionnaires de langue générale pour appuyer son analyse. Il est d'ailleurs intéressant de constater que les termes de l'audiovisuel et de la publicité qu'il a traités sont généralement recensés dans les dictionnaires de langue consultés, où ils portent la mention «recommandation officielle». Il ressort de l'enquête menée par Michel Chansou que seul un nombre très restreint des termes étudiés sont réellement bien implantés dans l'usage, constatation qui amène le chercheur à remettre en cause le mode de fonctionnement des commissions ministérielles de terminologie : «le système rigide et les dispositions réglementaires des arrêtés s'appliquent mal à la réalité de la langue et [...] ne tiennent pas suffisamment compte des conditions sociales de la communication» (p. 166).

La cinquième et dernière étude a été réalisée sous la direction de Daniel Gouadec par la section Terminologie et traitement automatique des données linguistiques du Centre de recherche sur les applications de l'informatique à l'enseignement de l'Université Rennes II. Elle porte sur 136 termes du domaine de l'informatique regroupés dans le Glossaire des termes officiels de l'informatique publié par la DGLF et parus dans les arrêtés de terminologie des 22 décembre 1981, 30 décembre 1983, 30 mars 1987 et 27 juin 1989. Il s'agit d'une étude fouillée, divisée en cinq volets distincts, chacun s'intéressant à un aspect particulier de la question. Il faut d'ailleurs souligner la remarquable précision avec laquelle Daniel Gouadec et ses collègues présentent les données et les résultats de leur enquête.

On a d'abord voulu attester l'emploi des termes officiels par le biais d'une recherche aléatoire dans un corpus écrit et un corpus oral. Les résultats obtenus ont permis de déterminer cinq indices de fréquence en fonction du nombre de documents dans lesquels l'emploi du terme avait pu être attesté. Les résultats de ce premier volet de l'analyse montrent clairement que les termes officiels de l'informatique apparaissent dans les sources consultées et qu'ils sont dans une grande majorité implantés. 
Le deuxième volet de l'étude s'intéresse aux corrélations entre les types de documents, de discours ou de communications et le vocabulaire utilisé. À la lumière des résultats obtenus, on constate que la présence des termes anglais varie selon les secteurs, le public et le type de documents concernés et que l'implantation des termes officiels, bien que généralisée, présente également des variations par secteurs, par types de documents et par types de publics.

Dans le troisième volet de l'étude, on a cherché à mesurer la connaissance des termes officiels et de leur signification chez 50 professionnels de l'informatique. Dans un premier questionnaire, on a demandé aux répondants de donner les termes correspondant à 20 définitions tirées des arrêtés de terminologie. Dans un deuxième questionnaire, on a soumis les répondants à la procédure inverse, c'est-à-dire qu'on leur a demandé de décrire ou de définir 20 termes officiels. Les résultats sont ici très clairs : les termes officiels sont en général mal connus des répondants.

Le quatrième volet de l'étude dirigée par Daniel Gouadec porte sur l'implantation des termes officiels dans les dictionnaires d'informatique. On a choisi 15 termes aux fins de l'analyse. Il ressort que les termes officiels n'apparaissent pas tous en entrée dans les dictionnaires français-anglais consultés, que l'intégration des termes en entrée dépend de leur fréquence d'emploi, que les termes officiels ne sont pas systématiquement cités comme équivalents dans les dictionnaires anglais-français et, finalement, qu'ils sont toujours accompagnés d'au moins une forme non officielle.

Finalement, dans le dernier volet de l'étude, on a recueilli par le biais d'un questionnaire l'opinion de 50 professionnels du domaine relativement à la francisation et à la normalisation du vocabulaire de l'informatique. Ici encore, les résultats sont fort intéressants. La majorité des répondants considèrent que l'anglais est une langue supérieure dans le domaine de l'informatique et que la francisation est ralentie, entre autres, par la globalisation des marchés, l'inertie et les habitudes, le retard dans la création de termes français et la production directe de communication en anglais. Par ailleurs, les répondants, qui de toute évidence ne connaissent pas les commissions de terminologie et encore moins les résultats de leurs travaux, estiment que la francisation relève d'abord et avant tout des entreprises, non des pouvoirs publics.

En guise de conclusion, on peut dire que bien que les méthodes adoptées et les résultats obtenus diffèrent d'une étude à l'autre, il est possible d'en tirer certaines conclusions générales. C'est d'ailleurs ce que nous propose Loïc Depecker dans son texte introductif, d'une limpidité et d'une précision dignes d'être soulignées. M. Depecker insiste notamment sur le fait que l'implantation terminologique doit se faire en fonction d'un ensemble de situations de communication et dans une certaine vision diachronique et que, comme le montrent trois des études, les données recueillies sont calculables à l'aide de variables précises de façon à fournir des résultats chiffrés et donc, à obtenir un indice d'implantation. En terminant, comme l'observe M. Depecker, «trop d'éléments entrent en jeu pour étayer véritablement un "jugement d'implantation" relativement fiable : aussi bien la nature des domaines, que les différences de spécialités, de situations de communication, d'interlocuteurs, de documents, etc. [...], échappent encore à l'analyse. Autant de données pour lesquelles il manque encore de grandes études d'implantation. Mais, et c'est une de leurs richesses, ces perspectives sont bien mises en valeur dans les présentes études. Et sans doute nous donnent-elles les pistes pour appréhender ce qu'il faudrait pour entrer davantage dans la problématique de l'implantation terminologique, et pour en faire un véritable instrument d'aménagement» (pp. XXXIV et XXXV).

ALINE FRANCEUR

Université de Montréal, Montréal, Canada 